The Other as Shamer scale - 2: Development and validation of a short version of a measure of external shame

Marcela Matos ${ }^{1 *}$ José Pinto-Gouveia ${ }^{1}$ Paul Gilbert ${ }^{2}$ Cristiana Duarte ${ }^{1}$ Cláudia Figueiredo ${ }^{3}$

${ }^{1}$ Cognitive-Behavioural Research Centre, University of Coimbra, Portugal

${ }^{2}$ Mental Health Research Unit, Kingsway Hospital, Derby UK

${ }^{3}$ CIDTFF, University of Aveiro, Portugal

* Correspondence concerning this article should be addressed to:

1

\begin{tabular}{|l|l}
\hline & 2 \\
\hline
\end{tabular}

1.

CINEICC, Faculdade de Psicologia e

Ciências da Educação, Universidade de

Coimbra

Rua do Colégio Novo, Apartado 6153

3001-802 Coimbra, Portugal

Telefone: $(+351) 239851450$

Fax: (+351) 239851462

E-mail: marcela.s.matos@gmail.com (M.

Matos)

2

Professor Paul Gilbert OBE

Mental Health Research Unit

Derbyshire Healthcare NHS Foundation

Trust,

Kingsway Hospital

Derby, DE22 3LZ

UK

p.gilbert@derby.ac.uk

\title{
Acknowledgments
}

This research has been supported by the first author (Marcela Matos) PostDoctoral grant number SFRH/BPD/84185/2012, sponsored by FCT (Portuguese Foundation for Science and Technology). 


\begin{abstract}
External shame arises from the perception of negative judgements about the self in the mind of others and is currently measured by Other As Shamer Scale (OAS). This scale has been used in numerous studies. This study sought to develop a valid and reliable shorter form of the scale, called OAS2, in an adult sample of 690 participants, using experts' item ratings and Confirmatory Factor Analysis. The OAS2 consisted of 8 items, which replicated the unidimensional structure of the OAS (Matos et al., 2011) and revealed a good fit. The OAS2 had good internal consistency (.82), similar to the longer version. The OAS2 has good concurrent and divergent validity, being highly correlated with the OAS $(r=.91)$. The OAS and OAS2 have very similar significant correlations with measures of internal shame, psychopathology and anger, with no significant difference between them. Our results, suggest that the OAS2 is an economic, valid and reliable measure of external shame.
\end{abstract}

Keywords: External shame; OAS; OAS2; Confirmatory factor analysis; Psychopathology

\title{
Highlights
}

- The OAS is a widely self-report instrument of external shame.

- A short version of the OAS was developed based on expert ratings of the OAS items.

- The 8-item OAS2 revealed a unidimensional structure and a good fit.

- The OAS2 had good internal consistency, concurrent and divergent validities.

- OAS2 is an economic, valid and reliable measure of external shame. 


\section{The Other as Shamer scale - 2: Development and validation of a short version of a measure of external shame}

\section{Introduction}

Shame is a major, self-conscious emotion that has significant impacts on people's sense of self, well-being and vulnerability to psychopathology (Gilbert, 1998; Kim, Thibodeau, \& Jorgensen, 2011; Tangney \& Dearing, 2002), including depression (Alexander, Brewin, Vearnals, Wolff, \& Leff, 1999; Cheung, Gilbert, \& Irons, 2004; Matos \& PintoGouveia, 2010), anxiety (Tangney, Wagner, \& Gramzow, 1992), paranoia (Matos, PintoGouveia, \& Gilbert, 2013), post-traumatic stress disorder (Harman \& Lee, 2010), eating disorders (Skarderud, 2007; Troop, Allan, Serpell, \& Treasure, 2008), and personality disorders (Rüsch et al., 2007).

However, Gilbert $(1998,2003)$ noted that conceptualizations of shame may differ between cultures. For example, in American, individualistic cultures, shame has become focused on inwardly directed self-focused attention and self-evaluation (Cook 1996; Tangney \& Dearing, 2002). Internal shame relates to a tendency to attend to the negative aspects of the self, to have global self-judgments of the self as bad, inferior and flawed (Gilbert, 1998; Tangney \& Dearing, 2002) and therefore to have hostile emotions associated with those judgments such as anger, contempt and even self-hatred (Whelton \& Greenberg, 2005). Internal shame is maintained through the process of self-criticism (Gilbert 2007). In contrast, there is a different tradition, focusing on how we experience ourselves in the minds of others. For example, the American sociologist Charles Cooley (1864-1929) famously developed the concept of the looking glass self and the degree to which we can feel shame when we see this disdain in the eyes of the other (see Cooley 1998). Jean Paul Sartre took the same view, that shame is very much about how we exist in the minds of the others. He wrote, "We often say 
that the shy man is embarrassed by his own body. Actually, this is incorrect; I cannot be embarrassed by my own body as I exist in it. It is my body as it is for the Other which embarrasses me" (cited in Mollon 1984, p. 212). Lewis (1992) sees the issue of exposure to 'the judging eyes of others' as central to the experience of shame. This attentional focus for shame has been called external shame and relates to a complex psychology of social attractiveness and monitoring how we exist for others and their judgments of us (Gilbert, $1998,2003,2007)$. Hence, the pain of external shame is rooted in the recognition that others view the self negatively. This form of shame is linked to a range of mental health difficulties (Kim et al., 2011), which arise because being seen negatively by others risks rejection, social criticism and ostracism, increases the risk of concealment and fear of discovery, and cuts individuals from potential validating, supportive signals/relations that stimulate positive affect and neurophysiological systems such as oxytocin and parasympathetic regulation (Cacioppo \& Patrick, 2008; Cozolino, 2007).

Both experiences of external and internalised shame can arise as an emotional reaction to specific events (i.e., episodic or state shame) but can also be associated with an elevated propensity to such reactions linked to trait-like qualities, especially metacognitive shame-focused beliefs (Tangney \& Dearing, 2002). There is also evidence that there can be gender differences with regard to shame, with women reporting higher levels of shame proneness (e.g. Benetti-McQuoid \& Bursik, 2005; Galhardo, Cunha, Pinto-Gouveia \& Matos, 2013).

\section{Measurement}

To explore the potential similarities and differences between an externally focused experience of shame and an internally focused experience, Allan, Gilbert and Goss, (1994) and Goss, Gilbert, and Allan, (1994) used items from a standardized Internalized Shame 
Scale (ISS; Cook, 1996) but changed the wording to offer statements that began "Other people see me as..." with descriptors such as "inadequate". So, the 18 items were changed such that "I see myself as being very small and insignificant" was modified into "Other people see me as small and insignificant". Thus, the focus shifted to how others see or judge the self. This self-report scale is designed to give an overall assessment of how people think they are seen by others and is therefore called the Other As Shamer Scale (OAS; Allan et al., 1994; Goss et al., 1994). The OAS is a trait metacognitive belief scale, and is currently a widely used self-report questionnaire measuring shame. There is now increasing evidence showing that this distinction between internal and external shame is valid and important and they relate to psychopathology in slightly different ways (Kim et al., 2011).

The original study of OAS' psychometric properties was derived from a sample of 156 undergraduate students (Goss et al., 1994). A Principal Component Analysis with varimax rotation revealed a three factor structure: "inferiority" accounting for $44 \%$ of the variance (eigenvalue of 7.92), "emptiness", accounting for $7.2 \%$ of the variance (eigenvalue of 1.28) and "how others behave when they see me making mistakes" accounting for $9.2 \%$ of the variance (eigenvalue of 1.66). Several items loaded on more than one factor or did not significantly load on any of the factors, sustaining the use of the OAS as a global measure of external shame, with a high internal consistency (Cronbach's alpha $=.92$ ). Regarding convergent validity, the OAS was found to be significantly associated with other shame measures, showing high correlations with shame trait measures, such as the ISS $(r=.81)$, and moderate correlations with state shame measures, such as the Dimensions of Conscience Questionnaire ( $r=.34$; Johnson et al., 1987) and the Adapted Dimensions of Conscience Questionnaire ( $r=.37$ to .46; ADCQ, Gilbert, Pehl, \& Allan, 1994). Furthermore, Allan et al. (1994) reported moderate to high correlations between the OAS and depressive symptoms ( $r$ 
$=.58$ to .73 , measured by Beck Depression Inventory; Beck, Steer, \& Garbin, 1988), anger at others and self ( $r=.38$ and .41, ADCQ, Gilbert, Pehl, \& Allan, 1994), and other clinically significant difficulties (e.g., severe depression $r=.47$, anxiety $r=.33$, somatic complaints $r=$ .18 , social dysfunctioning $r=.35$, assessed by the General Health Questionnaire; Goldberg \& Hillier, 1979).

Although the OAS scale has been consistently used across studies the research on its psychometric properties and adaptation to other populations is still scant. Therefore, Matos, Pinto-Gouveia, and Duarte (2011), recently investigated the OAS factor structure, internal consistency, test-retest reliability and convergent and divergent validly, in a large sample of the Portuguese general population $(N=811)$. Results showed that OAS is a valid and reliable measure of external shame. Principal Components Analysis results revealed a one factor structure, explaining $39.8 \%$ of the variance with excellent internal consistency (Cronbach's alpha $=.91)$ and temporal stability for a period of 4 to 6 weeks $(r=.70)$. Convergent and divergent validity was confirmed by the moderate correlations found with the Experience of Shame Scale (Andrews, Qian \& Valentine, 2002) and by the low to moderate correlations with Depression, Anxiety and Stress subscales (Lovibond, \& Lovibond, 1995).

\section{The current study}

Given the extensive evidence of the relevance of external shame for a wide range of mental health problems (Kim et al., 2011), we sought to create a shorter version of the OAS called OAS2 with the advantages of being easier and quicker to administer, especially in clinical settings, and thus placing less of a burden on respondents. Second, dropping items with lower correlations with the other items and with poor factor loadings could maximize the scale utility, without undermining its reliability (DeVellis, 2012).

Therefore, the aim of this study was to develop a short version of the OAS (OAS2) and examine its factorial structure through confirmatory procedures in a large sample of the 
general population. Additionally, this study explores whether a shorter scale version presents similar associations with psychopathology variables, to those of the original versions (Goss et al., 1994; Matos et al., 2011).

\section{Method}

Participants

Two research groups were used in the study. The first group were 14 clinical and research international experts who were asked to identify the top 10 items from the long OAS that best represented the construct of external shame. These items then became the basis for testing a model for the shorter version of shame in a larger population noted below. The experts were 9 female and 5 male and were deemed experts because of their theoretical and clinical knowledge on the evolutionary biopsychosocial model of shame (Gilbert, 1998, 2007).

Six hundred and ninety participants were second group to take part in this study, 312 $(45.2 \%)$ were students recruited from a public university and $378(54.8 \%)$ subjects recruited from the general community population in the center region of Portugal. Sixty five per cent of the participants were females $(n=449)$. The age range of the participants was $18-67(M=$ 30.38; $S D=12.33)$. The majority were single $(n=468 ; 67.8 \%)$ with $27.4 \%(n=189)$ married. In the general community sample, $23.6 \%(n=163)$ had middle class professions and $22.8 \%(n=157)$ had upper class professions. The participants' mean years of education was $14.32(S D=2.72)$. The data analysis treated the samples as one population because both the undergraduate students and the community sample and males and females showed similar means and standard deviations for the research variables.

\section{Measures}


Other As Shamer (OAS; Goss et al., 1994; Portuguese version used in the study by Matos et al., 2011) is a self-report instrument composed by 18 items measuring external shame. Respondents are asked to indicate the frequency on a 5 -point scale $(0=$ Never to $4=$ Almost Always) of their feelings and experiences to items such as, "I feel other people see me as not quite good enough". Higher scores reveal high external shame. In this study, OAS Cronbach's alpha was .89.

Experience of Shame Scale (ESS, Andrews et al., 2002) is a 27-item scale assessing three areas of (internal) shame: character shame (e.g., "Have you felt ashamed of the sort of person you are?"); behavioural shame (e.g., "Have you tried to cover up or conceal things you felt ashamed of having done?"); and body shame (e.g., "Have you felt ashamed of your body or any part of it?"). Each item indicates the frequency of experiencing, thinking and avoiding any of the three areas of shame in the past year and is rated on a 4-point Likert scale. Andrews et al. (2002) found this scale to have a high internal consistency (Cronbach's $\alpha=$ .92). In the present study, only the total of the ESS was used and showed a Cronbach's alpha of .94 .

Depression, Anxiety and Stress Scale (DASS-42; Lovibond \& Lovibond, 1995) is a selfreport measure composed of 42 items and designed to assess three dimensions of psychopathological symptoms: depression, anxiety and stress. Each item is rated on a 4-point scale. In the original version, subscales presented high internal consistency (Depression Cronbach's $\alpha=.91$; Anxiety $\alpha=.84$; Stress $\alpha=.90$ ). In the present study, the three subscales also showed high internal consistency ( $.92, .87, .92$ respectively). 
State-Trait Anger Expression Inventory (STAXI; Spielberger \& Sydeman, 1994) is a 44item self-report questionnaire assessing the intensity of anger as an emotional state (State Anger), and disposition to experience angry feelings as a personality trait (Trait Anger). STAXI comprises 5 scales (Anger-State, Anger-Trait, Anger-in, Anger-out, Anger Control) and 2 subscales (Anger Temperament and Angry Reaction). The items are rated in a 4-point scale. Internal consistency of the STAXI scales and subscales was high (.73 to .93). In the present study, only the Anger-State and Anger-Trait scales were used and showed high internal consistencies (.89 and .80 respectively).

\section{Procedure}

In order to generate the shorter version from the OAS two-step methodology was used. First, the series of self-report questionnaires described above were administered students to be completed at the end of a lecture, In the general population, a convenience sample was collected within the staff of institutions, namely schools and private corporations. Authorization from these institutions' boards was obtained and the self-report questionnaires were completed in the presence of the researcher. In line with ethical requirements, participants gave their informed consent after researchers explained the main aim of the study and emphasized that cooperation was voluntary and answers were confidential.

A second step was to ask the 14 experts to rate the OAS items. They were given a brief outline of external shame as representing a set of beliefs about how others may perceive us in terms of, for example, being looked down upon or being seen as inferior. They were then asked to choose, without hierarchical preference, the 10 items from the18-item OAS that better captured this idea of existing negatively in the minds of others, but to choose nonoverlapping items. The reason for this was to select a set of items that reflected the central 
theoretical components of external shame. Construct validity of the OAS2 was based on content validity resulting from the experts' ratings of items, followed by procedures for factorial validity, using confirmatory factorial analysis. In addition, the observation of reliability analysis indicators and convergent and divergent validity were considered for construct validity (DeVellis, 2012; Netemeyer, Bearden \& Sharma, 2003).

\section{Data analysis}

Statistical software IBM SPSS and IBM AMOS (v.20) was used to develop OAS2. The dimensionality analysis was assessed using confirmatory factor analysis. This procedure was chosen because it is one of the most robust and suitable to test underlying structures to latent variables, especially when these are unidimensional structures, such as the OAS (DeVellis, 2012; Kline, 2005). Internal reliability was examined using the Cronbach's alpha coefficient and item-total correlation. Pearson product-moment correlations were computed to assess the concurrent and divergent validities.

\section{Results}

Experts' rating of $O A S^{\prime}$ 'items

Table 1 presents the OAS original items ranked according to the experts' ratings. In order to obtain a shorter version of OAS we assumed a model with the top 8 items that were chosen by the majority of the experts ( $>$ than $57.1 \%$ ) as the most representative of the construct of external shame. This set of items represent overall negative evaluations about how others see and judge the self (e.g., not good enough, insignificant, unimportant, defective), thus capturing the concept of external shame as theoretically defined (Gilbert, 1998, 2007).

(Table_1_about_here) 


\section{OAS2 Confirmatory factor analysis}

In order to use Structural Equation modeling (SEM) in the data set assumptions regarding normality were checked for, in particular Kurtosis and outliers. In order to obtain acceptable results to proceed with the analyses, 3 multivariate outliers, that had the highest squared Mahalanobis distance (both $p<.001$ ), were excluded (Kline, 2005). Completed data from the participants was used.

We conducted a Confirmatory Factor Analysis (CFA) using a maximum likelihood estimator (ML) to test the adequacy of the 8-item OAS2 model and confirm the previous proposed OAS one-factor structure (Matos et al., 2011). The overview of the goodness-of-fit indices allowed us to consider this unifactorial model to have an acceptable global model fit [ $X^{2}(20)=113.271 ; p<.001 ; \mathrm{CMIN} / \mathrm{DF}=5.664 / \mathrm{GFI}=.960 / \mathrm{AGFI}=.929 / \mathrm{CFI}=.943 / \mathrm{TLI}=$ $.920 / \mathrm{RMSEA}=.082 / \mathrm{SRMR}=.044]$.

Regarding the estimates of observed variables (Table 2), standardized regression weights ranged from .46 (item 1$)$ to .78 (item 6 ) and all paths were statistically significant $(p<.001)$. The square multiple correlations $\left(R^{2}\right)$ indicate the reliability of the measure: item $1(.21)$ and item 6 (.61), have the lowest and the highest values, respectively. Therefore, all standardized indicators have theoretical and statistical support indicating that this is a plausible model for explaining the factorial structure of the OAS2.

The corrected item-total correlations showed adequate values that confirm the adequacy of these items to the construction of the measure and its internal consistency.

(Table_2_about_here)

Although items 1, 4 and 17 showed lower squared multiple correlations, the other local adjustment indices were good and their removal weakened the theoretical model and did not improve the scale reliability. 
In terms of the reliability of the OAS2, the Cronbach's alpha (.82) indicated a very good internal consistency. The similarity between this value and the one found for the OAS in this study $(\alpha=.89)$ supports the reliability of the OAS2 as a global measure of external shame.

\section{Gender differences}

Independent samples $t$ tests were conducted to explore gender differences in the original and short versions of the OAS and no significant differences were found (OAS: $t_{(685)}$ $=1.67, p=.095 ;$ OAS2: $\left.t_{(685)}=1.29, p=.199\right)$.

\section{OAS2 Concurrent and divergent validity}

To evaluate concurrent and divergent validity of the OAS2, Pearson product moment correlation coefficients were calculated between the OAS2 and OAS, ESS, DASS subscales and STAXI scales. The results given in Table 3 show that OAS2 was highly and positively correlated with the original OAS. Furthermore, OAS2 presented a strong and positive correlation with internal shame, as measured by the ESS. Regarding psychopathology indicators, OAS2 was moderately and positively associated with depressive and, to a lesser extent, anxiety and stress symptomatology. Also, OAS2 was positively and moderately related to state anger and, to a lesser extent, to trait anger. Such associations are similar in magnitude and directions to the ones found for the original OAS, supporting the concurrent and divergent validity of the short version of this scale.

$$
\text { (Table_3_about_here) }
$$

\section{Discussion}


There is now good evidence for the importance of external shame in the development and maintenance of a wide range of emotional and interpersonal problems (e.g., Cheung et al., 2004; Kim, et al., 2011; Matos \& Pinto-Gouveia, 2010; Matos, Pinto-Gouveia \& Gilbert, 2013). However, methodological advances in psychometric analyses point out the importance of considering both brevity and reliability when using self-report measures (DeVellis, 2012). Therefore, this study sought to develop and test the validity and reliability of a shorter version of the OAS, the OAS2, based on expert assessment of external shame items and then testing a model for this shorter 8-item version in a large heterogeneous sample of undergraduate students and participants from the general population.

Having developed the 8-item OAS2 confirmatory factor analysis was used to test the model fit to observed data metrics. This 8 -item shorter version fully replicated the one-factor structure of the original OAS (Matos et al., 2011) and corroborated the theoretical model of external shame (Gilbert, 1998, 2007). A significant chi-square goodness of fit index was found, as expected given the large sample size. Overall, the remaining goodness-of-fit indices indicated a good fit (Kline, 2005; Byrne, 2010). In addition, results showed that all standardized indicators had theoretical and statistical support and confirmed this as a plausible model to explain the factorial structure of the OAS2. Regarding reliability, the itemtotal correlations further confirmed the adequacy of these items. Finally, the OAS2 showed a very good internal consistency (.82), similar to that found for the OAS with this sample $(.89)$ and in its original (.92) (Goss et al., 1994) and previous studies (.91) (Matos et al., 2011). This added extra support for the reliability of the OAS2 as a shorter measure of external shame.

The concurrent and divergent validity analyses also corroborated our hypothesis with OAS2 correlating highly with the original version of the scale (Goss et al., 1994). Moreover, the OAS2 significantly correlated with internal shame and general psychopathology measures 
of depressive, anxiety and stress symptomatology, and with distinct dimensions of state and trait anger. Importantly, the magnitude and direction of the OAS2 correlations with these variables were similar, indeed almost identical (Table 3). In addition they were very similar to those found for the original OAS (Allan et al., 1994; Matos et al., 2011), thus supporting its concurrent and divergent validity regarding important psychopathological symptoms and emotional difficulties. These results suggest that individuals with high(er) levels of external shame, as measured by the OAS2, also experience feelings of internal shame, depressive, anxiety and stress symptomatology. OAS is also associated with anger. These data are in line with the empirical literature reporting a strong link between external shame and clinically significant indicators or difficulties (e.g., Cheung et al., 2004; Kim et al., 2011; Gilbert \& Miles, 2000; Matos \& Pinto-Gouveia, 2010; Troop et al., 2008).

To summarize, our findings extend the work on external shame measurement by confirming the adequacy of a shorter version of the OAS, which proved to be an economic, valid and reliable measure to assess external shame.

Nevertheless, some methodological limitations should be considered when interpreting these results. First, the use of a nonclinical sample impairs generalization to a clinical population. Thus, future studies could seek to replicate these findings using clinical samples, and explore the discriminant validity of the OAS2. Furthermore, although no gender differences were found in this study, future research could investigate the invariability and generalizability of the model in size equivalent samples of both genders. We also draw attention to the fact that the original OAS was sometimes shown to have different factors within it. Therefore future research may need to distinguish different forms of external shame; for example some people may feel external shame because they feel incompetent in certain ways or because of physical appearance or because of a history of trauma such as 
abuse. These different types of external shame might be different in terms of their link to psychopathology and the short version may not illuminate this.

Nonetheless, the OAS2 is a valid and reliable shorter measure of overall negative perceptions of how one thinks others see the self, offering an alternative to the OAS longer version. These findings may provide a relevant contribution to the assessment of shame and to research on the role external shame plays in human functioning and suffering. 


\section{References}

Alexander, B., Brewin, C., Vearnals, S., Wolff, G., \& Leff, J. (1999). An investigation of shame and guilt in a depressed sample. British Journal of Medical Psychology, 72, 323339. http://dx.doi.org/10.1348/000711299160031

Allan, S., Gilbert, P., \& Goss, K. (1994). An exploration of shame measures: II. Psychopathology. Personality and Individual Differences, 17, 719-722. http://dx.doi.org/10.1016/0191-8869(94)90150-3

Andrews, B., Qian, M., \& Valentine, J. (2002). Predicting depressive symptoms with a new measure of shame: The experience of shame scale. The British Journal of Clinical Psychology, 41, 29-42. http://dx.doi.org/10.1348/014466502163778

Benetti-McQuoid, J., \& Bursik, K. (2005). Individual differences in experiences of and responses to guilt and shame: Examining the lenses of gender and gender role. Sex Roles, 53(1-2), 133-142. http://dx.doi.org/10.1007/s11199-005-4287-4

Byrne, B. M. (2010). Structural equation modelling with amos: Basic concepts, applications, and programming. ( $2^{\text {nd }}$ ed.) New York, NY: Routledge Academic.

Cacioppo, J.T. \& Patrick, (2008) Loneliness: Human Nature and the Need for Social Connection. Norton: New York

Cheung, M., Gilbert, P., \& Irons, C. (2004). An exploration of shame, social rank and rumination in relation to depression. Personality and Individual Differences, 36, 11431153. http://dx.doi.org/10.1016/S0191-8869(03)00206-X

Cook, D. (1996). Empirical studies of shame and guilt: The Internalized Shame Scale. In D. L. Nathanson (Ed.). Knowing feeling. Affect, script and psychotherapy (pp. 132-165). New York, NY: W. W. Norton \& Company.

Cozolino, L. (2007). The neuroscience of human relationships: Attachment and the developing brain. New York: Norton 
DeVellis, R. (2012). Scale development: theory and applications ( $3^{\text {rd }}$ ed.). Thousand Oaks: Sage

Galhardo, A., Cunha, M., Pinto-Gouveia , J., \& Matos M. (2013). The Mediator Role of Emotion Regulation Processes on Infertility-Related Stress. Journal of Clinical Psychology in Medical Settings, 20, 497-507. http://dx.doi.org/10.1007/s10880-0139370-3

Gilbert, P. (1998). What is shame? Some core issues and controversies. In P. Gilbert and B. Andrews (Eds.). Shame: Interpersonal problems, psychopathology and culture (pp. 336). New York, NY: Oxford University Press.

Gilbert, P. (2003). Evolution, social roles and the differences in shame and guilt. Social Research, 70, 1205-1230.

Gilbert, P. (2007). The evolution of shame as a marker for relationship security. In J.L. Tracy, R.W. Robins \& J.P. Tangney (Eds.). The self-conscious emotions: Theory and research. (pp. 283-309). New York, NY: Guilford.

Gilbert, P. \& Miles, J. N. V. (2000). Sensitivity to social put-down: Its relationship to perceptions of social rank, shame, social anxiety, depression, anger and self-other blame. Personality and Individual Differences, 29, 757-774.

Gilbert, P., Pehl, J., \& Allan, S. (1994). The phenomenology of shame and guilt: An empirical investigation. British Journal of Medical Psychology, 67, 23-36. http://dx.doi.org/10.1111/j.2044-8341.1994.tb01768.x

Goss, K., Gilbert, P., \& Allan, S. (1994). An exploration of shame measures I. The "Other as Shamer Scale ". Personality and Individual Differences, 17, 713-717. http://dx.doi.org/10.1016/0191-8869(94)90149-X 
Harman, R. \& Lee, D. (2010). The role of shame and self-critical thinking in the development and maintenance of current threat in post-traumatic stress disorder. Clinical Psychology and Psychotherapy, 17(1), 13-24. http://dx.doi.org/10.1002/cpp.636

Johnson, R. C., Danko, G. P., Huang, Y. H., Park, J. Y., Johnson, S. B., \& Nagoshi, C. T. (1987). Guilt, shame and adjustment in three cultures. Personality and Individual Differences, 8, 357-364. http://dx.doi.org/10.1016/0191-8869(87)90036-5

Kim, S., Thibodeau, R., \& Jorgensen, R. (2011). Shame, guilt, and depressive symptoms: a meta-analytic review. Psychological Bulletin, 137(1), 68-96. http://dx.doi.org/10.1037/a0021466

Kline, R. (2005). Principles and practice of structural equation modeling (2th ed.). New York, NY: The Guilford Press.

Lovibond, P. \& Lovibond, H. (1995). The structure of negative emotional states: Comparison of the Depression Anxiety Stress Scales (DASS) with Beck Depressive and Anxiety Inventories. Behaviour Research and Therapy, 33(3), 335-343. http://dx.doi.org/10.1016/0005-7967(94)00075-U

Matos, M. \& Pinto-Gouveia, J. (2010). Shame as a traumatic memory. Clinical Psychology and Psychotherapy, 17 (4), 299-312. http://dx.doi.org/10.1002/cpp.659

Matos, M., Pinto-Gouveia, J., \& Duarte, C. (2011). Other as Shamer: Portuguese version and psychometric properties of an external shame measure. (Submitted manuscript).

Matos, M. Pinto-Gouveia, J., \& Gilbert, P. (2013). The effect of shame and shame memories on paranoid ideation and social anxiety. Clinical Psychology and Psychotherapy, 20, 334-349. http://dx.doi.org/10.1002/cpp.1766

Netemeyer, R., Bearden, W. \& Sharma, S. (2003). Scaling procedures: issues and applications. Thousand Oaks: Sage. 
Rüsch, N., Lieb, K., Göttler, I., Hermann, C., Schramm, E., Richter, H., Jacob, G.A., Corrigan, P. W., \& Bohus M. (2007). Shame and implicit self-concept in women with borderline personality disorder. American Journal of Psychiatry, 164, 500-508. http://dx.doi.org/10.1176/appi.ajp.164.3.500.

Skarderud, F. (2007). Shame and pride in anorexia nervosa: A qualitative descriptive study. European Eating Disorders Review, 15, 81-97. http://dx.doi.org/10.1002/erv.774

Spielberger, C. D. \& Sydeman, S. J. (1994). State-Trait Anger Inventory and State-Trait Anger Expression Inventory. In M. E. Maurice (Ed.), The use of psychological testing for treatment planning and outcomes assessment (pp. 300-321). New Jersey, NJ: Lawrence Erlbaum Associates.

SPSS Inc. Released 2009. PASW Statistics for Windows, Version 18.0. Chicago, IL: SPSS Inc.

Tangney J. \& Dearing R. (2002). Shame and guilt. New York, NY: Guilford Press.

Tangney, J., Wagner, P., \& Gramzow (1992). Proneness to shame, proneness to guilt and psychopathology. Journal of Abnormal Psychology, 101, 469-478. http://dx.doi.org/10.1037//0021-843X.101.3.469

Troop, N. A., Allan, S., Serpell, L., \& Treasure, J. L. (2008). Shame in women with a history of eating disorders. European Eating Disorders Review, 16, 480-488. http://dx.doi.org/10.1002/erv.858.

Whelton, W.J \& Greenberg, L.S (2005). Emotion in self-criticism. Personality and Individual Differences, 38, 1583-1595. 
Table 1. Experts' rating of OAS items

\begin{tabular}{|c|c|c|c|}
\hline Rank & Item & Frequency & $\%$ \\
\hline 1 & 1. I feel other people see me as not good enough. & 13 & 92.9 \\
\hline 2 & 6. Other people see me as small and insignificant. & 13 & 92.9 \\
\hline 3 & 8. People see me as unimportant compared to others. & 13 & 92.9 \\
\hline 4 & 5. Other people see me as not measuring up to them. & 12 & 85.7 \\
\hline 5 & 2. I think that other people look down on me. & 11 & 78.6 \\
\hline 6 & 4. I feel insecure about others opinions of me. & 10 & 71.4 \\
\hline 7 & 17. Others think there is something missing in me. & 10 & 71.4 \\
\hline 8 & 7. Other people see me as somehow defective as a person. & 8 & 57.1 \\
\hline 9 & 11. I think others are able to see my defects. & 7 & 50 \\
\hline 10 & 14. Other people always remember my mistakes. & 7 & 50 \\
\hline 11 & 15. Others see me as fragile. & 7 & 50 \\
\hline 12 & 3. Other people put me down a lot. & 5 & 35.7 \\
\hline 13 & 16. Others see me as empty and unfulfilled. & 5 & 35.7 \\
\hline 14 & $\begin{array}{l}\text { 10. People see me as striving for perfection but being unable to reach my own } \\
\text { standards. }\end{array}$ & 4 & 28.6 \\
\hline 15 & 12. Others are critical or punishing when I make a mistake. & 4 & 28.6 \\
\hline 16 & 9. Other people look for my faults. & 3 & 21.4 \\
\hline 17 & 13. People distance themselves from me when I make mistakes. & 3 & 21.4 \\
\hline \multirow[t]{2}{*}{18} & 18. Other people think I have lost control over my body and feelings. & 3 & 21.4 \\
\hline & Total & 14 & \\
\hline
\end{tabular}

Note. In bold are the items selected for the OAS2 CFA 
Table 2. Standardized Regression Weights, Squared Multiple Correlations $\left(R^{2}\right)$ and Item-Total Correlations for the items considered in the model

\begin{tabular}{|c|c|c|c|}
\hline Items & $\begin{array}{c}\text { Standardized Regression } \\
\text { Weight }\end{array}$ & $R^{2}$ & $\begin{array}{l}\text { Item-Total } \\
\text { Correlation }\end{array}$ \\
\hline OAS2_1. I think that other people look down on me. & .463 & .214 & .451 \\
\hline OAS2_6. People distance themselves from me when I make mistakes & .783 & .613 & .588 \\
\hline OAS2_8. Others think there is something missing in me. & .722 & .522 & .472 \\
\hline OAS2_5. People see me as unimportant compared to others. & .705 & .497 & .623 \\
\hline OAS2_2. Other people see me as not measuring up to them. & .664 & .441 & .673 \\
\hline OAS2_4. Other people see me as somehow defective as a person. & .497 & .247 & .488 \\
\hline OAS2_17. Others think there is something missing in me. & .505 & .255 & .645 \\
\hline OAS2_7. Others see me as empty and unfulfilled. & .577 & .333 & .467 \\
\hline
\end{tabular}


Table 3. Means (M), standard deviations (SD) and product-moment correlation coefficients between OAS2 and OAS and internal shame (ESS), Depression, anxiety and stress symptomatology (DASS-42) and anger $(\mathrm{STAXI})(N=687)$

\begin{tabular}{ccccc}
\hline & $M$ & $S D$ & OAS2 & OAS \\
\hline OAS2 & 7.29 & 3.88 & - & - \\
OAS & 18.46 & 7.85 & $.91^{* *}$ & - \\
ESS & 48.30 & 13.06 & $.54^{* *}$ & $.48^{* *}$ \\
Depression & 5.27 & 5.71 & $.49^{* *}$ & $.50^{* *}$ \\
Anxiety & 5.19 & 5.11 & $.35^{* *}$ & $.39^{* *}$ \\
Stress & 10.76 & 6.82 & $.36^{* *}$ & $.35^{* *}$ \\
Trait-Anger & 19.22 & 4.50 & $.27^{* *}$ & $.28^{* *}$ \\
State-Anger & 11.80 & 3.64 & $.31^{* *}$ & $.35^{* *}$ \\
\hline
\end{tabular}

$* p<.010 . * * p<.001$

Key. OAS, External shame-original measure; OAS2, External shame-short measure; ESS, Internal shame. 